\title{
Surfactant containing Si-MCM-41: An efficient basic catalyst for the Knoevenagel condensation
}

\author{
Leandro Martins ${ }^{\mathrm{a}}$, Tito J. Bonagamba ${ }^{\mathrm{b}}$, Eduardo R. de Azevedo ${ }^{\mathrm{b}}$, \\ Pascal Bargiela ${ }^{\mathrm{c}}$, Dilson Cardoso ${ }^{\mathrm{a}, *}$ \\ ${ }^{a}$ Chemical Engineering Department, Federal University of São Carlos, Rodovia Washington Luís, \\ km 235, 13565-905 São Carlos, SP, Brazil \\ ${ }^{\mathrm{b}}$ Instituto de Física de São Carlos, Universidade de São Paulo, P.O. Box 369, 13560-970 São Carlos, SP, Brazil \\ ${ }^{\mathrm{c}}$ Instituto de Química, Universidade Federal da Bahia, R. Barão de Geremoabo s/n, 40170-290 Salvador, BA, Brazil \\ Received 3 March 2006; received in revised form 17 June 2006; accepted 19 June 2006 \\ Available online 2 August 2006
}

\begin{abstract}
Si-MCM-41 molecular sieves, which are largely used in a number of acid and redox catalyzed reactions, as reported by the literature, were used as basic catalyst for the Knoevenagel condensation. This reaction involves the condensation of carbonyl compounds with active methylene compounds to generate unsaturated products. The catalyst, which pores were occluded by the organic template, provided high activity in mild conditions, even under $10^{\circ} \mathrm{C}$. The high activity is due to the presence of siloxy anions, of high basicity, located in the pore cavity of the hexagonally ordered mesopores. Ion exchange procedures evidenced the presence of these high basic sites as a consequence of the high $\mathrm{pH}$ increase of the solution containing tetramethylammonium chloride, or the high $\mathrm{H}^{+}$consumption, added to a the medium as $\mathrm{HCl}$, to keep constant $\mathrm{pH}$. O 1s XPS and ${ }^{29} \mathrm{Si} \mathrm{CP} / \mathrm{MAS}$ NMR measurements confirmed the existence of only $\equiv \mathrm{SiO}^{-} \mathrm{CTA}^{+}$ionic pair, which is the basic site itself.

(C) 2006 Elsevier B.V. All rights reserved.
\end{abstract}

Keywords: Si-MCM-41; Basic catalysis; Knoevenagel condensation; Molecular sieves

\section{Introduction}

Catalysis from solids with acid properties, when compared to other solids containing basic sites have received, along the years, large attention as a result of the significance these catalysts have in petroleum refinement. However, in many reactions, basic catalysts may be used to replace acids with a selectivity gain, because they inhibit the development of other parallel reactions, such as cracking and coke formation [1].

The use of microporous solids with high aluminum content such as zeolite $13 \times$ as basic catalysts is widely known [2,3]. Contrarily, materials with highly ordered pores in the mesopores region containing silicon and oxygen in their chemical composition have been little studied. These materials are called MMS (mesoporous molecular sieves) and were first developed in the 1990s by Mobil Company [4,5]. They have a

\footnotetext{
* Corresponding author. Fax: +55 1633518266.

E-mail address: dilsoncardoso@ power.ufscar.br (D. Cardoso).
}

pore diameter ranging from 2 to $50 \mathrm{~nm}$ and a surface area in the range of $1000 \mathrm{~m}^{2} \mathrm{~g}^{-1}$. These new materials have provided the development of new catalysts with larger dispersion in the active area, a characteristic that is critical for the improvement of current industrial processes [6].

A way to develop basicity in mesoporous materials is by dispersing alkali metal oxides in the channel. Kloetstra et al. [7] initially proposed to disperse cesium oxide particles in $\mathrm{Si}$ MCM-41 pores. For the preparation, the molecular sieve is impregnated with a concentrated cesium acetate solution followed by calcination so that the acetate ion can decompose. The generated basic sites were evaluated by a programmed $\mathrm{CO}_{2}$ desorption and showed a high basic strength. However, due to the treatments used throughout the preparation, its structure was extensively damaged, as seen through X-ray diffraction and by nitrogen adsorption isotherms [7]. It is widely known that $\mathrm{Si}-\mathrm{O}$ bonds, present in these materials, hydrolyze in high $\mathrm{pH}$ environments. Because mesoporous molecular sieves have high surface area, the hydrolysis in these bonds takes place more easily, even in a moderately basic environment. Results show 
that $\mathrm{pH} 8.5$ is the upper limit value to prevent the MCM-41 mesopore structure from undergoing significant destruction [8].

Another way to get basic Si-MCM-41 sites is by functionalizing its surface with organic compounds, particularly with those containing terminal amines in their composition $[9,10]$. Before functionalizing, the ionic surfactant present in the pores is removed by calcination, generating silanol groups, which are anchoring points. Concentration of silanol groups depends on how the ionic surfactant is removed. Calcined mesoporous silica has lower concentration of silanol groups as a result from their high condensation temperatures. If the surfactant is removed by extraction with a solvent, their pore surface is kept with plenty of silanol groups, with nearly $100 \%$ coverage [9].

All these procedures, which are used to generate basic sites on Si-MCM-41, are hard to process and not always lead to the planned basic sites, stable enough for catalysis application. Recently, Kubota et al. [11,12] achieved excellent results during Knoevenagel condensation by using Si-MCM-41 molecular sieve while keeping the surfactant inside the pores called [CTA]Si-MCM-41. In this catalyst, active sites are high basicity $\equiv \mathrm{SiO}^{-}$sites, which are in the channels. But, despite good results, there is no further information available on the acid-basic properties of Kubota's catalyst.

This work is designed to study the chemical behavior of catalyst [CTA]Si-MCM- 41 by using it in the Knoevenagel condensation model reaction in mild conditions.

\section{Experimental}

\subsection{Synthesis of [CTA]Si-MCM-41 molecular sieve}

[CTA]Si-MCM-41 catalyst was synthesized through Cheng et al. method [13]. Samples were prepared from aerosil silica, cetyltrimethylammonium bromide (CTABr) and tetramethylammonium hydroxide (TMAOH). Composition of the reaction medium was $\mathrm{SiO}_{2}$ : 0.27, CTABr: 0.19, TMAOH: $40 \mathrm{H}_{2} \mathrm{O}$.

First, $9.22 \mathrm{~g}$ TMAOH$\cdot 5 \mathrm{H}_{2} \mathrm{O}$ (Aldrich) and $26.35 \mathrm{~g} \mathrm{CTABr}$ (Vetec) were diluted in $188.35 \mathrm{~g}$ deionized water. Then, the mixture was kept stirring at $60{ }^{\circ} \mathrm{C}$ until a clear solution was achieved. After cooling the solution to room temperature, $16.09 \mathrm{~g}$ of a silica source was added. The sample was aged for $24 \mathrm{~h}$ before going to thermal treatment, which was carried out in autoclaves under autogenous pressure at $150{ }^{\circ} \mathrm{C}$ for 2 days. Finally, the sample was filtered, washed successively times with deionized water up to $\mathrm{pH}<10$ and dried at $60{ }^{\circ} \mathrm{C}$ for $24 \mathrm{~h}$. Solid yield was approximately $10 \%$ compared to the gel mass.

To compare with [CTA]Si-MCM-41, the surfactant occluded in the pores was removed through calcination, under an oxygen flow, through a heating ramp of $1{ }^{\circ} \mathrm{C} / \mathrm{min}$ up to $520^{\circ} \mathrm{C}$, kept subsequently at this temperature for $6 \mathrm{~h}$.

Basic properties of the [CTA]Si-MCM-41 molecular sieve were established via titration with a hydrochloridric acid solution $\left(0.1 \mathrm{~mol} \mathrm{~L}^{-1}\right)$ from a suspension containing $1.5 \mathrm{~g}$ [CTA]Si-MCM-41 in $200 \mathrm{~mL}$ deionized water or $0.5 \mathrm{~mol} \mathrm{~L}^{-1}$ of TMACl or $\mathrm{NaCl}$ solution. Preliminary assays showed that when kept in an aqueous suspension, $\mathrm{pH}$ raised quickly. As a result, throughout titration, a constant $\mathrm{pH}$ value of 7.0 was kept by continuously adding acid. The consumption of protons/g solid was achieved by recording the volume of the acid source consumed throughout the titration period.

\subsection{Characterization}

Small angle X-ray diffraction patterns were achieved with a diffractometer D-5000 (Siemens), using powder method at $1.4^{\circ}<2 \theta<10^{\circ}$ interval. $\mathrm{Cu} \mathrm{K} \alpha(40 \mathrm{kV}$ and $40 \mathrm{~mA})$ radiation was used, nickel filter and $0.6 \%$ min speed.

Nitrogen adsorption/desorption isotherms were achieved with an equipment supplied by Quantachrome (Nova-1200). Before analysis, $50 \mathrm{mg}$ of the sample, calcined or assynthesized, was vacuum treated at $110^{\circ} \mathrm{C}$ for $2 \mathrm{~h}$. Surface areas were estimated through BET equation [14] and the pore diameters were established via BJH method [15].

Percent of organic material present in Si-MCM-41 molecular sieves, as-synthesized or acid titrated, was established via $\mathrm{C}, \mathrm{H}$ and $\mathrm{N}$ analyses in a Fisons equipment, model EA1108-CHNS-O.

NMR analyses were performed at a VARIAN unit INOVA spectrometer operating at 79.5 and $400 \mathrm{MHz}$ for ${ }^{29} \mathrm{Si}$ and ${ }^{1} \mathrm{H}$, respectively. Magic-angle spinning at $5 \mathrm{kHz}$ was used in all experiments. In order to obtain more quantitative spectra a cross-polarization excitation scheme using a radiofrequency ramp (ramp CP/MAS) was used [16]. The ramp CP/MAS spectra were obtained with $5 \mathrm{~ms}$ of contact time and recycle delay of $2 \mathrm{~s} .{ }^{29} \mathrm{Si}$ direct polarization experiments (DP/MAS) were performed using a $5-\mathrm{ms} \pi / 2$ excitation pulse and $30 \mathrm{~s}$ of recycle delay. In all experiments, during the $20-\mathrm{ms}$ acquisition time a $70 \mathrm{kHz}{ }^{1} \mathrm{H}$ broadband two-pulse phase-modulation (TPPM) [17] decoupling scheme was used.

$\mathrm{X}$-ray photoelectron spectroscopy measurements were done in equipment ESCALAB MKII de VG, source $\mathrm{Al} \mathrm{K \alpha}$, under pressure of $10^{-8}$ mbar. C $1 \mathrm{~s}$ was taken as reference at $285 \mathrm{eV}$.

\subsection{Knoevenagel condensation}

This reaction was performed in a $20 \mathrm{~mL}$ using an initial reactants concentration of $0.5 \mathrm{~mol} \mathrm{~L}^{-1}$ in the following way: $1.07 \mathrm{~g}$ (10 mmol) benzaldehyde (Acros); $1.14 \mathrm{~g}$ (10 mmol) ethyl cyanoacetate (Acros) and $15.42 \mathrm{~g}(17.9 \mathrm{~mL})$ toluene (Merck) as solvent were added to a borosicalate glass reactor equipped with magnetic stirring and temperature control. In order to prevent from the presence of benzoic acid in the reaction media (oxidation of benzaldehyde) fresh reactant was used. Additionally, chromatographic data revealed that, under the mild reaction conditions used in this work, benzoic acid is not present. The reaction was carried out at temperature intervals from 10 to $50{ }^{\circ} \mathrm{C}$ and atmospheric pressure. As soon as the reaction medium achieved planned temperature, $1 \mathrm{~g}$ of the catalyst was added. Two different catalysts were evaluated in this study: (i) as-synthesized MCM-41, without any additional treatment and (ii) as-synthesized MCM-41 titrated with $\mathrm{HCl}$. After $6 \mathrm{~h}$ reaction, the catalyst was retrieved for analysis and reuse by centrifuging and wash out with benzene to remove 
adsorbed products. Analysis of reactants and products in liquid samples was carried out with a Varian chromatograph equipped with DB-1 capillary column and a flame ionization detector (FID). Other analysis conditions included: injector temperature, $210{ }^{\circ} \mathrm{C}$; column temperature, $140{ }^{\circ} \mathrm{C}$ for $12 \mathrm{~min}$ and $230{ }^{\circ} \mathrm{C}$ for $15 \mathrm{~min}$, and $\mathrm{He}$ as carrier gas.

\section{Results and discussion}

Si-MCM-41 samples, calcined or not, showed a typical diffractogram (Fig. 1a). As-synthesized sample showed a specific area of $1.0 \mathrm{~m}^{2} \mathrm{~g}^{-1}$ (Table 1) while calcined sample showed a specific area of $990 \mathrm{~m}^{2} \mathrm{~g}^{-1}$. Elemental C, H and $\mathrm{N}$ analysis of as-synthesized $\mathrm{Si}-\mathrm{MCM}-41$ sample showed a $\mathrm{C} / \mathrm{N}$ ratio close to 19 , which corresponds to the CTA chemical composition, indicating that tetramethylammonium hydroxide, the mineralizing agent used in the synthesis, is not included in the solid phase.

\subsection{Ion exchange of [CTA]Si-MCM-41 molecular sieve}

Despite [CTA]Si-MCM-41 pores are completely filled with the surfactant agent, Kubota et al. [11,12] reported that its activity in Knoevenagel condensation is extremely high, even in mild conditions. With the objective to develop even further the activity of these materials, by increasing the contact of the reactants with active sites (larger surface area), the catalyst [CTA]Si-MCM-41 was subjected to an ion exchange process with an organic cation with a lower volume than $\mathrm{CTA}^{+}$, i.e. tetramethylammonium $\left(\mathrm{TMA}^{+}\right)$. Removal of the surfactant occluded in Si-MCM-41 pores by ion exchange has been performed by Hitz and Prins, by extraction with a hydrochloridric acid solution in ethanol [18].

In this study, during the ion exchange with a TMACl aqueous solution, a sudden $\mathrm{pH}$ rise was evidenced. Essays with different TMACl concentrations showed a relation between TMA cation concentration and the suspension $\mathrm{pH}$, which was measured 10 min after the mixture was completed (Fig. 2), that is, for higher TMA concentration, higher $\mathrm{OH}^{-}$concentration.

To understand and better quantify this behavior, experiments were performed in a TMACl solution, by keeping $\mathrm{pH}$ at 7.0 throughout the ionic exchange, by adding diluted $\mathrm{HCl}$ to the

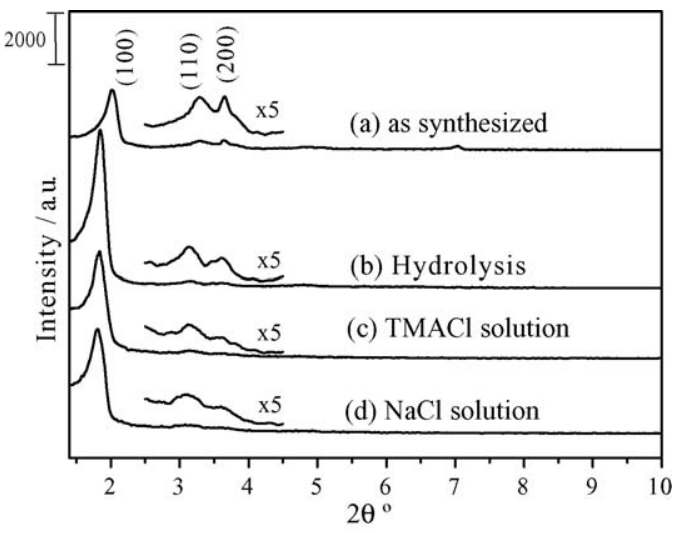

Fig. 1. X-ray diphratograma of mesoporous silica: (a) synthesized and (b)-(d) exchanged in an aqueous medium while keeping $\mathrm{pH}$ at 7.

suspension. This exchange takes place very quickly: while $1.5 \mathrm{~g}$ of the sample is added to $200 \mathrm{~mL}$ of $0.5 \mathrm{~mol} \mathrm{~L}^{-1} \mathrm{TMACl}$ solution, $\mathrm{pH}$ rises to readings close to 10 , with the need to frequently add $\mathrm{HCl}$ to keep $\mathrm{pH}$ at 7.0. Curve 1 in Fig. 3 shows how $\mathrm{H}^{+} / \mathrm{g}$ [CTA]Si-MCM-41 consumption changed as a function of time in the presence of TMA cation, where it can be seen the quick $\mathrm{H}^{+}$consumption.

Once the sample was washed with deionized water more than 10 times and chemical analysis of the mesoporous material indicated that it did not contain residual TMAOH, results from curve 1 in Fig. 3 suggest that surface $\equiv \mathrm{SiO}^{-}$sites, with high basicity, are responsible for $\mathrm{pH}$ increase as a result of proton consumption from water. In this process (Eqs. (1) and (2)) formation $\equiv \mathrm{SiOH}$ groups takes place and partial channel unblocking as a result from CTA removal:

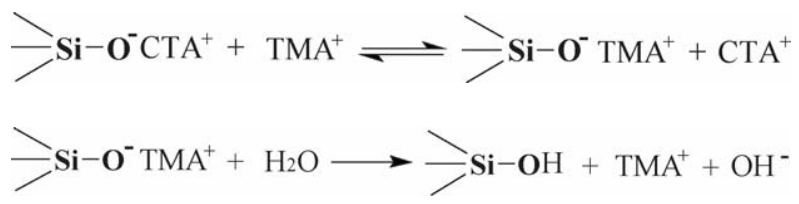

The presence of a considerable concentration of TMA cation in the aqueous medium, forces CTA cation to exit the pores (Eq. (1)), and the ion exchange takes place. However, because $\equiv \mathrm{SiOH}$ groups are more stable than $\equiv$ SiOTMA groups, this cation is quickly changed by a proton (Eq. (2)), with subsequent

Table 1

Modified MCM-41 molecular sieve composition

\begin{tabular}{|c|c|c|c|c|c|c|}
\hline Sample & CTA/Si & $\left(\mathrm{CTA}^{+}+\mathrm{H}^{+}\right)^{\mathrm{a}} / \mathrm{Si}$ & BET area $\left(\mathrm{m}^{2} \mathrm{~g}^{-1}\right)$ & $V_{\mathrm{p}}(\mathrm{mL} / \mathrm{g})$ & $a_{\mathrm{H}}(\AA)$ & $D_{\mathrm{p}}(\AA)$ \\
\hline $\mathrm{MCM} 41$, original $^{\mathrm{b}}$ & 0.16 & $0.16 \pm 0.01$ & 1 & 0 & 51.0 & 0 \\
\hline MCM-41, hydrolysis ${ }^{c}$ & $0.10(37.5 \%)^{\mathrm{d}}$ & $0.14 \pm 0.01$ & 314 & $0.25(25.2 \%)^{\mathrm{e}}$ & 55.4 & 35.1 \\
\hline $\mathrm{MCM}^{-41, \mathrm{TMACl}^{\mathrm{f}}}$ & $0.08(50.0 \%)$ & $0.16 \pm 0.01$ & 374 & $0.28(28.3 \%)$ & 56.1 & 35.5 \\
\hline $\mathrm{MCM}-41, \mathrm{NaCl}^{\mathrm{f}}$ & $0.07(56.2 \%)$ & $0.14 \pm 0.01$ & 385 & $0.30(30.3 \%)$ & 57.0 & 35.6 \\
\hline MCM-41, calcined ${ }^{\mathrm{g}}$ & $0(100.0 \%)$ & - & 990 & $0.99(100.0 \%)$ & 46.4 & 33.8 \\
\hline
\end{tabular}

${ }^{a}$ CTA molar quantity present in the solid. Protons consumed in the titration.

b $\mathrm{C} / \mathrm{N}$ ratio $=19.5$.

c Sample taken by simple hydrolysis, according to Eq. (3).

d In parenthesis: molar percent of removed CTA.

e In parenthesis: volume of available pores.

f Sample taken by ion exchange with TMACl or $\mathrm{NaCl}$.

g Sample taken through original MCM-41 calcination. 


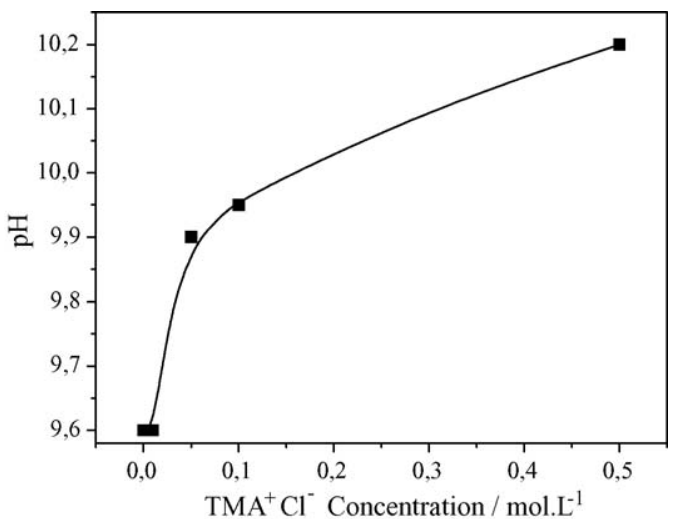

Fig. 2. Ion exchange without $\mathrm{pH}$ control $10 \mathrm{~min}$ after startup: suspension $\mathrm{pH}$ as a function of tetramethylammonium chloride concentration.

release of $\mathrm{OH}^{-}$. This quick proton consumption (Eq. (2)) can also be explained by the fact that the TMA cation, as opposed to CTA, provides high mobility in a reaction medium because of its smaller volume and because it is not included among the micelles, as it happens with CTA. Therefore, Eqs. (1) and (2) explain why $\mathrm{pH}$ increases as TMA cation concentration increases (Fig. 2). As TMA concentration increases, the ionic strength in the solution is higher, leading to larger TMA exchange (Eq. (1)). When no cation is added to the solution, only hydrolysis can be seen (Eq. (3)), and proton consumption speed, to maintain $\mathrm{pH}$ at 7.0, is smaller, as shown in curve 3 of Fig. 3:

$$
\underset{\mathrm{Si}}{-} \mathbf{O}^{-} \mathrm{CTA}^{+}+\mathrm{H}_{2} \mathrm{O} \leftrightarrows \frac{\searrow}{\leftrightarrows} \mathrm{Si}-\mathbf{O H}+\mathrm{CTA}^{+}+\mathrm{OH}^{-}
$$

To determine the quantity of cation involved in the ion exchange, an exchange procedure was performed in a $0.5 \mathrm{~mol} \mathrm{~L}^{-1} \mathrm{NaCl}$ solution. Similarly to TMA cation, the presence of sodium in the liquid phase also increased the consumption speed of $\mathrm{H}^{+}$(curve 2 of Fig. 3), compared to simple hydrolysis. Exchange speed was a little higher in the presence of sodium cation, because it has a smaller volume and therefore diffuses more easily.

After $24 \mathrm{~h}$ of ion exchange, the amount of sodium present in the solid (determined by elemental chemical analysis) was

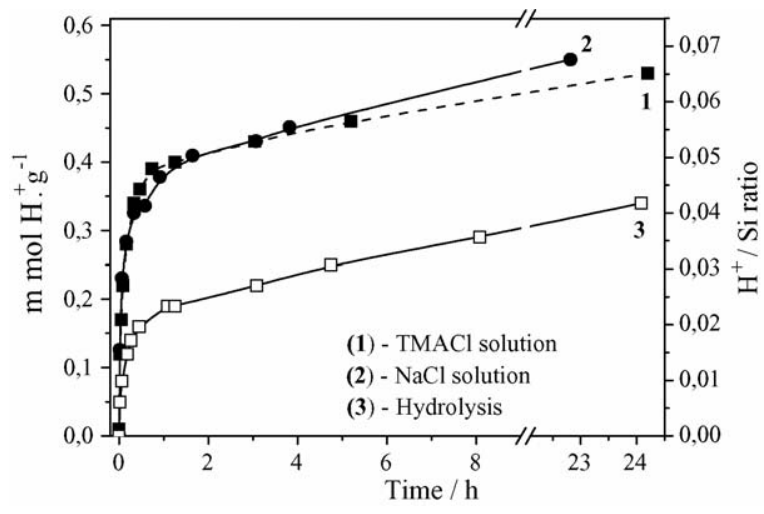

Fig. 3. Proton consumption as a function of time throughout [CTA]Si-MCM-41 sample titration, with $\mathrm{pH}$ being controlled at 7.0. approximately $5 \%$ of that expected, when taking into account the quantity of CTA exchanged $(\mathrm{Na} / \mathrm{Si}=0.005)$. This result confirms that despite most sodium is not in the solid, in $\equiv \mathrm{SiONa}$ form, its presence in the solution helps to develop the hydroxide ion, similarly to that showed in Eq. (2). Such behavior confirms the hypothesis based on Eqs. (1)-(3), indicating the presence of high basicity $\equiv \mathrm{SiO}^{-}$groups. Cation molar balance at the end of the $24-\mathrm{h}$ period showed that the ratio $\left(\mathrm{CTA}^{+}+\mathrm{H}_{\text {consumed }}{ }^{+}\right) / \mathrm{Si}$ is kept at approximately 0.15 , close to the original value of 0.16 (Table 1 ). This result confirms that for each removed $\mathrm{CTA}^{+}$a silanol group was created in the solid. The ratio $\mathrm{C} / \mathrm{N}$ equal to 18.5 achieved in the sample treated in a $\mathrm{TMACl}$ containing solution suggests that, just as it happens with sodium, only a small amount of TMA cation remains in the solid as $\equiv$ SiOTMA. These results show that the initial objective to develop even further the activity of these materials, by increasing the contact of the reactants with active sites (三SiOTMA) cannot be achieved.

Fig. 1 shows X-ray diffractogram from as-synthesized as well exchanged MCM-41 by titration procedures at $\mathrm{pH} 7.0$, indicating that the mesoporous material structure is kept throughout the exchange.

CTA/Si results shown in Table 1 indicate that approximately $50 \%$ of the CTA cation present in the original MCM-41 was exchanged by submitting it to a TMACl solution at $\mathrm{pH}$ 7.0. However, the mesoporous volume built during the exchange with TMACl is very small, compared to calcined MCM-41 (approximately 28\%) as shown in the nitrogen physisorption isotherm (Fig. 4 and Table 1). The reason for such behavior could be explained, for example, by a preferential exchange of the CTA present on the external surface of MCM-41 particles. Exchanging CTA for a cation of lower volume would not increase significantly the pore volume, because the external surface of the particles does not significantly help this parameter. But this hypothesis is unlikely to be true because CTA cations present in the external surface of the particles are poorly connected to $\mathrm{SiO}^{-}$groups and, therefore, are easily leached during washing process. A second hypothesis to explain the origin of the pore volume could be the removal of

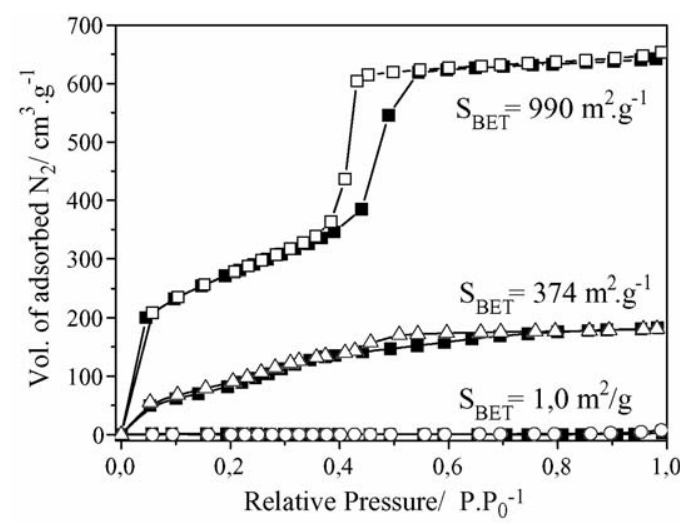

Fig. 4. Nitrogen physisorption isotherms of MCM-41 molecular sieves. Filled points: adsorption and empty points: dessorption. ( $\square$ ) and ( $\square$ ) Calcined MCM-

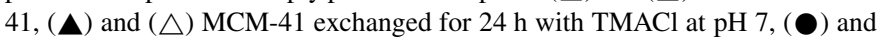
(O) MCM-41 as synthesized. 

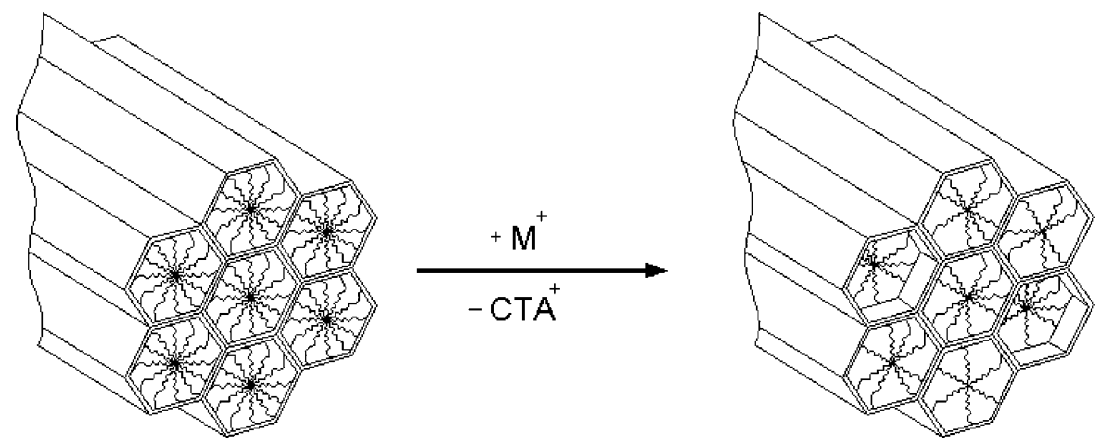

Fig. 5. Ion exchange process proposed between an $\mathrm{M}^{+}$cation and CTA ${ }^{+}$cation in sample [CTA]Si-MCM-41.

CTA cation progressively to the interior of the channels, but in such case, both properties (CTA extraction and pore volume) should be proportional. A third and more likely hypothesis would be the uneven exchange of these cations along the channels, as depicted in Fig. 5. In this case, the CTA extraction not necessarily provides accessibility to the pore.

Results shown in Table 1 also shows that, as reported by other authors [13], throughout calcination, there is a strong contraction of the mesoporous hexagonal arrangement parameter $\left(a_{\mathrm{H}}\right)$, as a result from the condensation of neighboring silanol groups. Results also show that after a partial ion exchange of the $\mathrm{CTA}^{+}$with $\mathrm{H}^{+}$, the mesoporous hexagonal arrangement parameter and the diameter of the pores $\left(D_{\mathrm{p}}\right)$ in the molecular sieves tend to increase. Such behavior may result from lower CTA concentration, whose presence may contract the pore diameter, as a result from its interaction to micelle formation.

\subsection{Knoevenagel condensation in toluene}

Results from Knoevenagel condensation (Eq. (4)) between benzaldehyde (1) and ethyl cyanoacetate (2) to form ethyl-2cyano-3-phenylacrylate (3) are shown in Fig. 6. [CTA]SiMCM-41 catalyst had high activity, even in temperatures that were very below the ones reported by other authors [2]. Throughout the reaction, the presence of a single product was always evidenced (3) and the ratio between the consumed

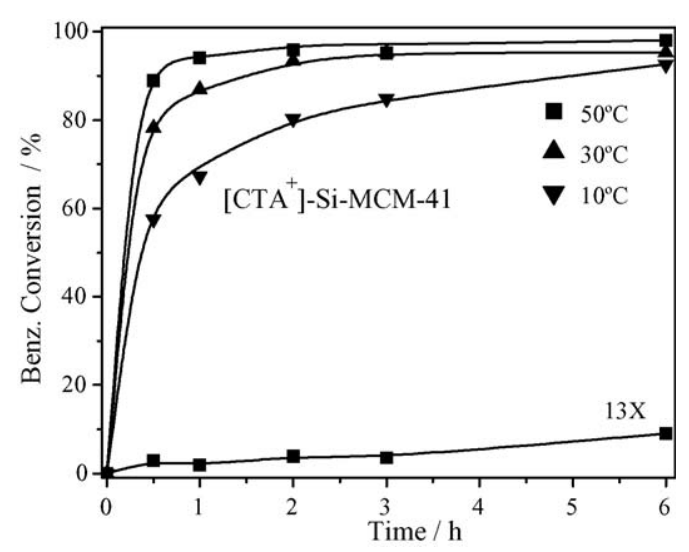

Fig. 6. Benzaldehyde conversion as a function of time for different reaction temperatures. reactants ( $\mathbf{1}$ and $\mathbf{2}$ ) around unity, indicated that the subsequent Michael addition reaction (Eq. (5)) does not take place at the temperatures here used:

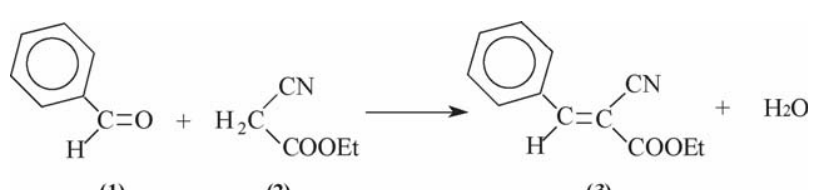

(1)

(2)

(3)

$\Delta \mathrm{G}_{298 \mathrm{~K}}=-98,7 \mathrm{KJ} \cdot \mathrm{mol}^{-1}$

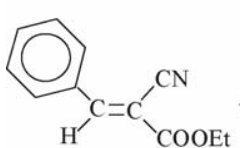

(3)<smiles>CCOC(=O)CC#N</smiles>

(2)

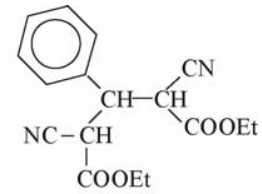

$\Delta \mathrm{G}_{298 \mathrm{~K}}=-19,3 \mathrm{KJ} \cdot \mathrm{mol}^{-1}$

The high reactant consumption speed, in aprotic medium, confirms the presence of high basicity, as noticed during ion exchange studies in aqueous media (Fig. 3). When calcined SiMCM-41 was added to the reaction medium, a very low conversion was seen $(\sim 2 \%)$, suggesting that this material has very weak basic sites, possibility due to residual cations, which are not completely removed. Even molecular sieve $13 \times$, which has been extensively studied as a basic catalyst for the Knoevenagel condensation [2], showed a low conversion at $50{ }^{\circ} \mathrm{C}$ ( $\sim 9 \%$ after 6 h, Fig. 6$)$.

Fig. 7a shows benzaldehyde conversion as a function of time for the catalyst reused in up to four successive batches. Its activity is slightly reduced after every new use, probably as a result from the leaching of part of the CTA to the reaction medium (step E in Fig. 8). This result will be better discussed further.

Results from thermogravimetry shown in Fig. 9a and Table 2 indicate that CTA leaching to the reaction medium, in the first use of a catalyst, is only ca. $0.3 \% \mathrm{~m} / \mathrm{m}$. The derivate curve resulting from the loss of mass in as-synthesized [CTA]SiMCM-41 sample (Fig. 9b) provides a signal at approximately $160{ }^{\circ} \mathrm{C}$ (indicated by an arrow). This signal is related to CTA decomposition taking place at pore-mouth $[11,12,19]$. For samples used from the third catalytic test, no presence of this signal was seen, thus confirming that partial CTA leaching from 

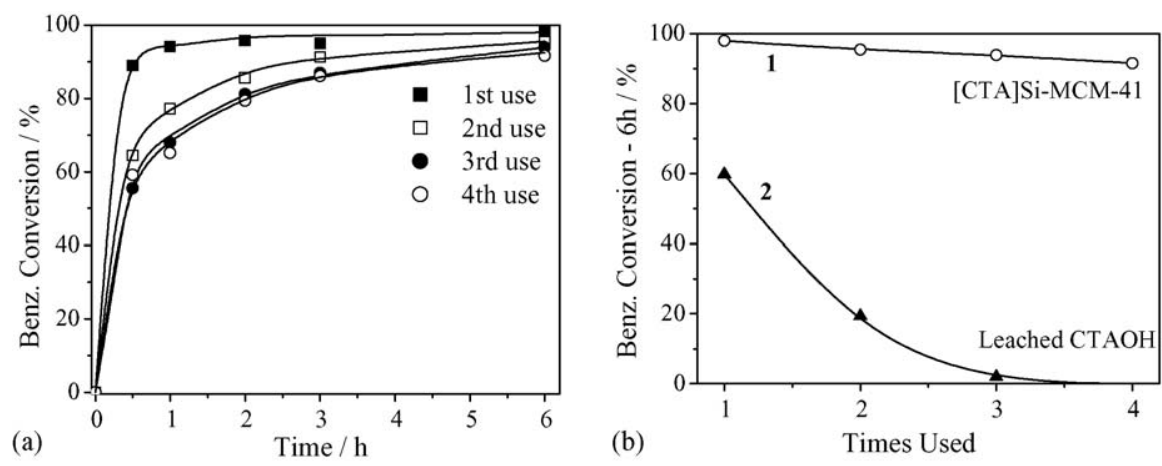

Fig. 7. (a) Benzaldehyde conversion as a function of time and (b) after $6 \mathrm{~h}$ for [CTA]Si-MCM- 41 catalyst reused in four successive cycles. Reaction temperature $=50^{\circ} \mathrm{C}$.

pore-mouth to the reaction medium tends to reduce as the catalyst is used. These cations are probably the ones most weakly connected to the micelles and their leaching, therefore, could be one of the responsible for the slight reduction of the catalyst activity, as shown in Fig. 7b, curve 1.

To verify if the Knoevenagel reaction also occurred in homogeneous phase, the liquid phase resulting from a catalytic test was isolated from the as-synthesized solid catalyst and new reactants were added to the filtrated. The new mixture was submitted to the same conditions as in the presence of the solid catalyst. The results are in curve 2 of Fig. $7 \mathrm{~b}$ and shows that, despite low quantity of leached CTA, by utilizing the filtrated from the catalyst used for the first time, a significant catalytic activity, but smaller than curve 1 , can be seen. When the filtrated from the second reaction is used, a much smaller activity is seen, thus confirming that smaller $\mathrm{CTA}^{+}$quantity is leached to the

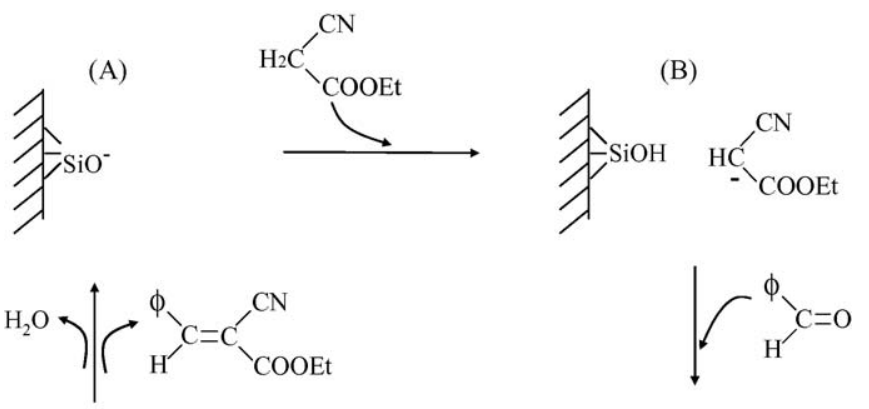

(D)

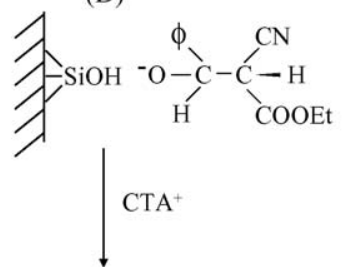

(E)
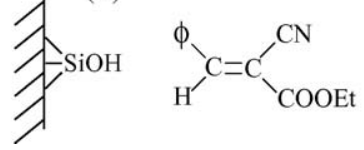

$\mathrm{CTA}^{+}{ }^{-} \mathrm{OH}(l)$

Fig. 8. Probable benzaldehyde condensation steps with ethyl cyanoacetate using [CTA]Si-MCM-41 catalyst. reaction medium. Finally, the third filtrate has practically no activity. These results differ from that of Kubota et al. [11,12], which found no activity in the homogeneous phase, because they washed the catalyst exhaustively with hot water, before using it.

Curiously, despite the fact that in the third use of the catalyst, no activity was found in the homogeneous phase, the activity of the solid remains practically the same (Fig. 7b, curves 2 and 1 , respectively). This suggests that, after the third use of the catalyst, no more leaching of CTA occurs. The explanation for this stabilization can be achieved taking into account the hypothesis of Kubota et al. [11], that the losses of active sites occurs mainly at pore-mouth, similarly as shown in Fig. 5. The remaining CTA, at the interior of the pore, are not so easily leached because they are more stable, as a consequence of a higher interaction of the non-polar tails, as stated by Li et al. [20] and Pool and Bolhuis [21].

The catalyst behavior in heterogeneous as well in homogeneous phase, shown in Fig. $7 \mathrm{~b}$ can be explained through the scheme represented in Fig. 8. The steps A-C are similar to those proposed by Corma et al. [22], for Knoevenagel reactions using zeolites. Depending on the stability of the CTA cation in the micelle, the hydroxide ion eliminated from the alkoxide ion (step D) can react in two different ways: forming water and regenerating the siloxy active site (step A), or forming CTAOH in the liquid phase.

Fig. 10 shows benzaldehyde conversion as a function of time by using $100 \mathrm{mg}$ of catalysts obtained at the end of the titration with $\mathrm{HCl}$ (about 24 h, Fig. 3). It is significant to see that despite the CTA content in exchanged samples being not very different among them (Table 1), the catalytic activities are. The slightly lower activity seen in the sample subjected to hydrolysis only (curve 1 respect to 2 ) is possibly due to the need for the reactants to diffuse to reach active sites $\equiv$ SiOCTA, which are deeper inside the pores than in the original sample (curve 1). For samples titrated in a $\mathrm{TMACl}$ or $\mathrm{NaCl}$ solutions (curves 3 and 4) the catalyst activity is much lower than previous ones and, therefore, another unknown factor should prevail. The activity calculated on a $\mathrm{Si}$ mol basis leads to the same conclusions. It is necessary to mention that despite the samples used in curves 2-4 (Fig. 10) have a much higher surface area than the as-synthesized used in curve 1 (see Table 1), their activities are lower. 

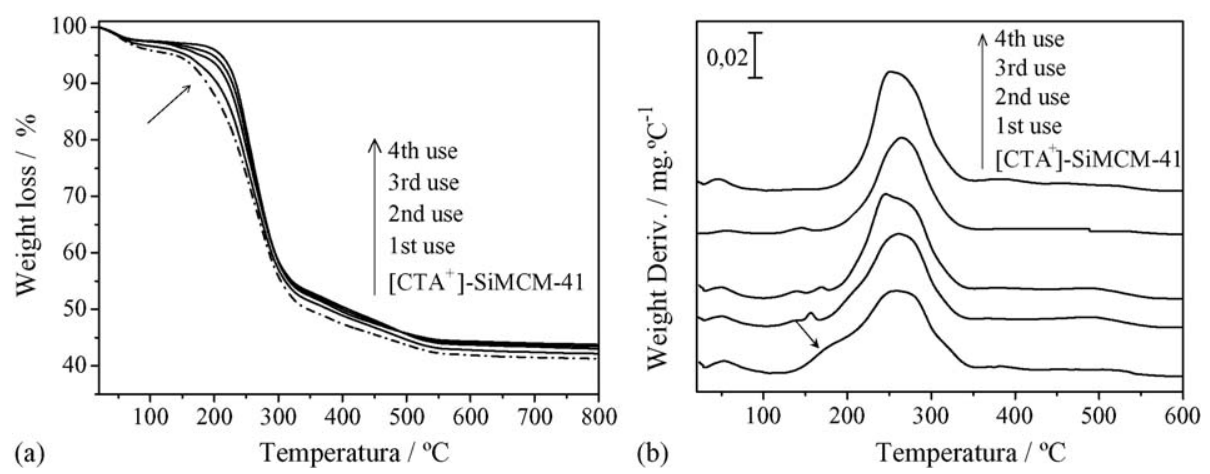

Fig. 9. (a) Thermogravimetric analysis and (b) derivate curves resulting from the loss of mass for [CTA]Si-MCM-41 catalyst before and after its use.

In addition to higher stability of active sites in exchanged samples, measurements for X-ray diffraction show that the catalyst structure is kept intact after its use. Therefore, the micelles present in the channels of the catalyst are significant for the mesopore structure to be kept as planned, both during the exchange (Fig. 3) and the reaction.

\subsection{Siloxy group characterization: ${ }^{29}$ Si MAS NMR and $O$ 1s/XPS spectroscopy}

Solid-state ${ }^{29} \mathrm{Si}$ nuclear magnetic resonance experiments $\left({ }^{29} \mathrm{Si} \mathrm{NMR}\right)$ employing magic-angle spinning (MAS) with direct or cross polarization (DP or CP) has been shown to be a reliable mean to characterize various silicate materials [23]. Particularly, CP process is interesting, thus it allows discriminating against ${ }^{29} \mathrm{Si}$ nuclei bonded close to hydrogen atoms [24]. By ${ }^{29} \mathrm{Si}$ NMR spectroscopy, the fraction of Si atoms bonded to $n \mathrm{Si}$ atoms, identified as $Q^{n}$ species $(0 \leq n \leq 4)$, as well bonded to single and geminal $\mathrm{SiOH}$ groups, $Q^{3}$ and $Q^{2}$ silicon sites, respectively, can be discriminated sensitively [2427].

Fig. $11 \mathrm{a}$ and b, as well as Table 3 show ${ }^{29} \mathrm{Si}$ DP and CP/MAS NMR spectra of as-synthesized and calcined MCM-41 samples. For both samples three peaks are observed, at $-91,-100$ and $109 \mathrm{ppm}$. According to Sindorf and Maciel [26], the low intensity peak at $-91 \mathrm{ppm}$ in calcined samples corresponds to silicon atoms bonded to two siloxane bonds $\mathrm{Si}-\mathrm{O}-\mathrm{Si}-\left(Q^{2}\right)$ and two geminal silanol groups. Similarly, the resonance peak at $-100 \mathrm{ppm}$ is attributed to silicon atoms with three siloxane bonds $\left(Q^{3}\right)$ and one silanol. Finally, the resonance peak at $-109 \mathrm{ppm}$ is related to silicon atoms with four siloxane bonds $\left(Q^{4}\right)$.

Table 2

Loss of mass seen in the thermogravimmetric analysis

\begin{tabular}{ll}
\hline Sample & Loss of mass $(\%)^{\mathrm{a}}$ \\
\hline [CTA]SiMCM-41 & 54.2 \\
First use & 53.9 \\
Second use & 53.6 \\
Third use & 53.5 \\
Fourth use & 53.5 \\
\hline
\end{tabular}

\footnotetext{
${ }^{\text {a }}$ At temperatures ranging from 120 to $800{ }^{\circ} \mathrm{C}$.
}

For as-synthesized, [CTA]Si-MCM-41 sample, almost no difference between DP/ and CP/MAS NMR spectra is observed, as well in $Q^{3} /\left(Q^{2}+Q^{4}\right)$ ratio $(51.9-50.4 \%$, Table 3$)$. This is a strong indication that the siloxy groups, $\mathrm{SiO}^{-} \mathrm{M}^{+}$, are not hydrogen-bonded, but they are present major as $\mathrm{SiO}^{-} \mathrm{CTA}^{+}$ ionic pairs, generating $\mathrm{SiO}^{-}$basic sites. Because there is a longer distance between ${ }^{29} \mathrm{Si}$ in the lattice and ${ }^{1} \mathrm{H}$ nuclei belonging to $\mathrm{CTA}^{+}$chain and since the ${ }^{1} \mathrm{H}$ nuclei in $\mathrm{CTA}^{+}$are rather mobile (as observed in the ${ }^{1} \mathrm{H}$ spectra, not shown), the $\mathrm{CP}$ transfer between these nuclei is not very effective. This makes the contribution of the $\mathrm{SiO}^{-} \mathrm{CTA}^{+}$ionic pair to the $Q^{3}$ signal similar in both CP/MAS and DP/MAS spectra [26]. In contrast, there is a very large difference between DP/ and CP/MAS spectra in the calcined sample, especially in the $Q^{3}$ species (see Fig. 11) and as well in $Q^{3} /\left(Q^{2}+Q^{4}\right)$ ratio $(36.3-65.5 \%$, Table 3). Because silanol groups are expected to exhibit a very effective ${ }^{1} \mathrm{H}^{2}{ }^{29} \mathrm{Si} \mathrm{CP}$ transfer, the higher intensity of the peak at $-100 \mathrm{ppm}$ in the CP/MAS as compared with the DP/MAS spectra suggests the presence of a very high concentration of silanol groups in the calcined sample, not observed in the noncalcined one.

Additionally, in literature is well known that O 1s XPS is a way of directly determining Lewis basicity in zeolites and related aluminosilicates materials [28,29] and that, small variations in oxygen binding energy means great difference in structural oxygen basicity [30,31].

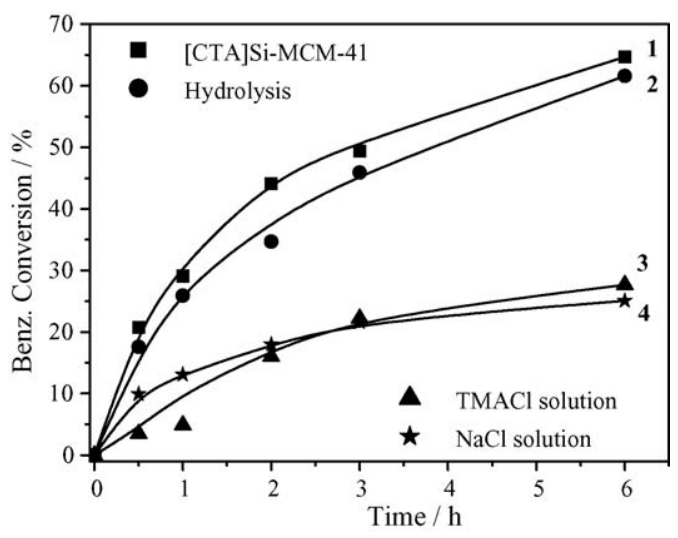

Fig. 10. Benzaldehyde conversion as a function of time at $50{ }^{\circ} \mathrm{C}$ for catalysts modified by ion exchange (using $100 \mathrm{mg}$ mass). 

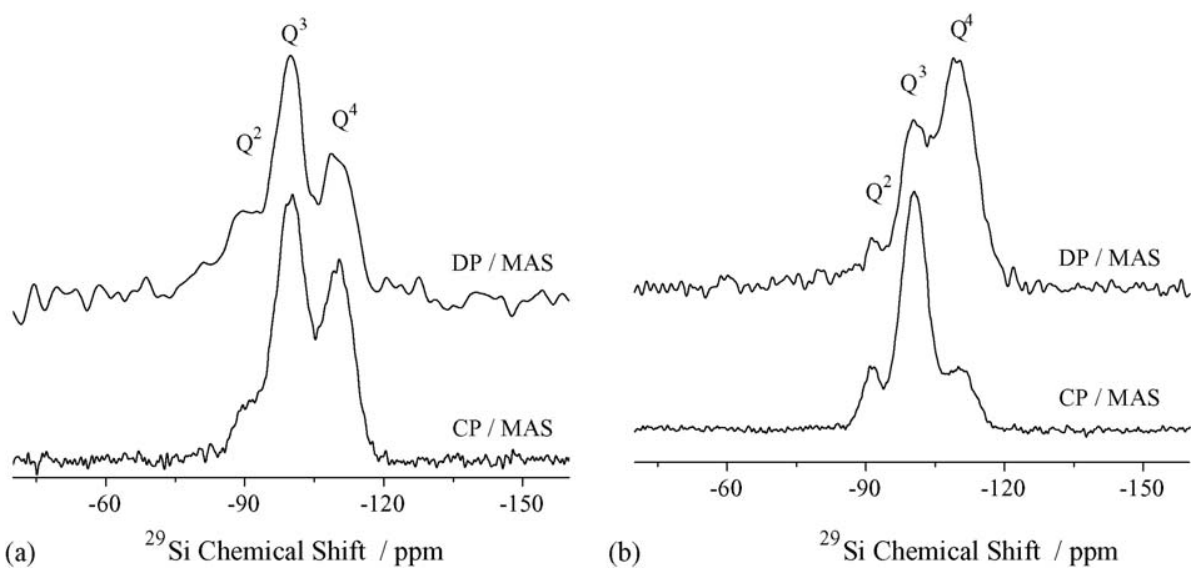

Fig. 11. ${ }^{29} \mathrm{Si} \mathrm{DP} /$ and CP/MAS NMR spectra for (a) as-synthesized and (b) calcined MCM-41.

Table 3

Relative peak areas (\%) in the ${ }^{29} \mathrm{Si} \mathrm{DP} /$ and CP/MAS NMR spectra of MCM-41 as-synthesized and calcined

\begin{tabular}{llllll}
\hline Sample & Signal & $Q^{2}(\%)$ & $Q^{3}(\%)$ & $Q^{4}(\%)$ & $Q^{3} /\left(Q^{2}+Q^{4}\right)$ \\
\hline [CTA]Si-MCM-41 & DP & 17.7 & 51.9 & 30.4 & $1.1 \pm 0.1$ \\
& CP & 11.6 & 50.4 & 38.0 & $1.0 \pm 0.1$ \\
Si-MCM-41 calcined & DP & 15.7 & 36.3 & 48.0 & $0.6 \pm 0.1$ \\
& CP & 18.0 & 65.5 & 16.5 & $1.9 \pm 0.1$ \\
\hline
\end{tabular}

O 1s XPS measurements of as-synthesized and calcined MCM-41, shown in Fig. 12a and b, respectively, indicate that binding energy of $\mathrm{O} 1 \mathrm{~s}$ is much lower in presence of the voluminous CTA cation $(532.5 \mathrm{eV})$, which means that the Lewis basicity of the $\mathrm{Si}-\mathrm{O}$ oxygen atom, belonging to [CTA]SiMCM-41 sample, is much higher. Similarly, Li et al. [32] studied the influence of Ba content in MCM-41 basic catalysts and found that the binding energy of $\mathrm{O} 1 \mathrm{~s}$ decreases from 533.0 to $532.5 \mathrm{eV}$ as the $\mathrm{Ba}$ content increases. Consequently, as $\mathrm{CTA}^{+}$ is a voluminous cation, its interaction with $\equiv \mathrm{SiO}^{-}$sites is weak, moving the chemical balance $\mathrm{SiOCTA} \leftrightarrow \mathrm{SiO}^{-}+\mathrm{CTA}^{+}$to the right and increasing $\equiv \mathrm{SiO}^{-}$basicity. Because the [CTA]SiMCM-41 catalyst channels are filled with the surfactant, according to Kubota et al. [11,12], the high activity seen in Knoevenagel condensation is mostly due to the basic sites present in the pore-mouth.

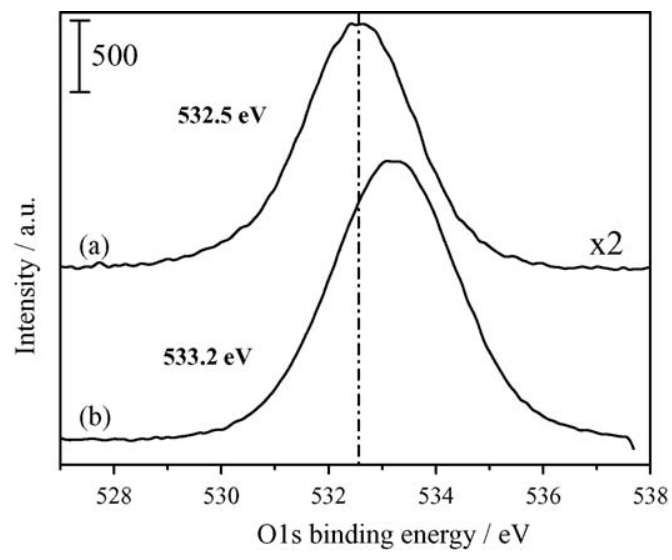

Fig. 12. O 1s XPS spectra of (a) as-synthesized and (b) calcined MCM-41.
Additionally to these results, measurements of ${ }^{29} \mathrm{Si}$ DP/MAS NMR, performed by Kubota et al. [11] in as-synthesized [CTA]Si-MCM-41, showed two peaks $Q^{3}$ and $Q^{4}$, more than $50 \%$ in the form of $Q^{3}$, attributed to the presence of $\mathrm{Si}(3-\mathrm{OSi}, 1-$ $\mathrm{O}^{-}$) moieties. Therefore, these authors indirectly suggested that $\equiv \mathrm{SiO}^{-}$sites are the ones responsible for the high basic activity of this catalyst. This proposal was also presented by Brunel et al. [33], that using XPS suggested the existence of $\mathrm{SiO}^{-} \mathrm{H}_{3} \mathrm{~N}^{+} \mathrm{R}$ ionic pairs in MCM-41 containing grafted amine groups. In both works, the authors suggest the existence of $\equiv \mathrm{SiO}^{-} \mathrm{M}^{+}$groups, where $\mathrm{M}^{+}$(instead of $\mathrm{H}^{+}$) is $\mathrm{CTA}^{+}$or an organic cation, by indirect means and differently of the methods employed in this work.

\section{Conclusions}

Mesoporous silica Si-MCM-41, with its pores still filled with the surfactant cation provided strong $\equiv \mathrm{SiO}^{-}$basic sites. The high catalyst activity in toluene and low temperatures was evidenced by the conversion of $99 \%$ of the reactants in a short reaction period. Despite this molecular sieve not showing shape selectivity property, as the reaction takes place at the poremouth only, it showed to be a very promising basic catalyst for fine chemistry synthesis.

Ion exchange in aqueous media at a neutral and constant $\mathrm{pH}$ evidenced the presence of very strong $\equiv \mathrm{SiO}^{-}$basic sites. To keep pH constant throughout the exchange, there was a need to continuously add hydrochloridric acid. Although the contribution to the basicity due to $\mathrm{CTA}^{+} \mathrm{OH}^{-}$ion pair, occluded insided of the MCM-41 channels, cannot be excluded, the existence of siloxy basic sites was supported by O 1 s XPS and ${ }^{29} \mathrm{Si}$ CP/MAS NMR measurements.

Due to the presence of part of the CTA remaining inside the channels, becoming part of the micelles, no change was seen in the X-ray diffraction pattern of the samples, both in the ion exchange and in the reaction.

\section{Acknowledgements}

The authors would like to thank Heloise O. Pastore (IQ/ Unicamp) for taking DRX measurements and Sonia Maria 
Cabral de Menezes (Cenpes/Petrobras) for suggestions about ${ }^{29} \mathrm{Si}$ NMR experiments. The Brazilian research agencies FAPESP and CNPq supported this project.

\section{References}

[1] K. Tanabe, W.F. Hölderich, Appl. Catal. A: Gen. 181 (1999) 399.

[2] U.D. Joshi, P.N. Joshi, S.S. Tamhankar, V.V. Joshi, C.V. Rode, V.P. Shiralkar, Appl. Catal. A: Gen. 239 (2003) 209.

[3] D. Barthomeuf, Micropor. Mesopor. Mater. 66 (2003) 1.

[4] J. Weitkamp, M. Hunger, U. Rymsa, Micropor. Mesopor. Mater. 48 (2001) 255.

[5] J.S. Beck, J.C. Vartuli, W.J. Roth, M.E. Leonowicz, C.T. Kresge, K.D. Schmitt, C.T.-W. Chu, D.H. Olson, E.W. Sheppard, S.B. McCullen, J.B. Higgins, J.L. Schlenker, J. Am. Chem. Soc. 114 (1992) 10834.

[6] H. Van Bekkum, E.M. Flanigen, P.A. Jacobs, J.C. Jansen, Introduction to Zeolite Science and Practice, Elsevier, 2001.

[7] K.R. Kloetstra, M. van Laren, H. van Bekkum, J. Chem. Soc., Faraday Trans. 93 (1997) 1211.

[8] C.N. Pérez, E. Moreno, C.A. Henriques, S. Valange, Z. Gabelica, J.L.F. Monteiro, Micropor. Mesopor. Mater. 41 (2000) 137.

[9] C.-M. Yang, K.-J. Chao, J. Chin. Chem. Soc. 49 (2002) 883.

[10] F. Fajula, D. Brunel, Micropor. Mesopor. Mater. 48 (2001) 119.

[11] Y. Kubota, Y. Nishizaki, H. Ikeya, M. Saeki, T. Hida, S. Kawazu, M. Yoshida, H. Fujii, Y. Sugi, Micropor. Mesopor. Mater. 70 (2004) 135.

[12] Y. Kubota, H. Ikeya, Y. Sugi, T. Yamada, T. Tatsumi, J. Mol. Catal. A: Chem. 249 (2006) 181.

[13] C.F. Cheng, D.H. Park, J. Klinowski, J. Chem. Soc., Faraday Trans. 93 (1997) 193.
[14] S. Brunauer, P.H. Emmet, E. Teller, J. Am. Chem. Soc. 60 (1938) 309.

[15] E.P. Barrett, L.G. Joyner, P.P. Halenda, J. Am. Chem. Soc. 73 (1951) 373.

[16] O.B. Peersen, X.L. Wu, I. Kustanovich, S.O. Smith, J. Magn. Res., Ser. A 104 (1993) 334.

[17] A.E. Bennett, C.M. Rienstra, M. Auger, K.V. Lakshmi, R.G. Griffin, J. Chem. Phys. 103 (1995) 6951.

[18] S. Hitz, R. Prins, J. Catal. 168 (1997) 194.

[19] Y. Kubota, Y. Nishizaki, H. Ikeya, J. Nagaya, Y. Sugi, Stud. Surf. Sci. Catal. 141 (2002) 553.

[20] Z.H. Li, S.J. Roy, Y.Q. Zou, R.S. Bowman, Environ. Sci. Technol. 32 (1998) 2628.

[21] R. Pool, P.G. Bolhuis, J. Phys. Chem. B 109 (2005) 6650.

[22] A. Corma, V. Fornés, R.M. Martín-Aranda, H. García, J. Primo, Appl. Catal. 59 (1990) 237.

[23] S. Haukka, E.L. Lakomma, A. Root, J. Phys. Chem. 97 (1993) 5085.

[24] X.S. Zhao, G.Q. Lu, A.K. Whittaker, G.J. Millar, H.Y. Zhu, J. Phys. Chem. B 101 (1997) 6525.

[25] C. Cannas, M. Casu, A. Musinu, G. Piccaluga, J. Non-Cryst. Solids 351 (2005) 3476.

[26] X.S. Zhao, G.Q. Lu, J. Phys. Chem. B 102 (1998) 1556.

[27] D.W. Sindorf, G.E. Maciel, J. Am. Chem. Soc. 103 (1981) 4263

[28] M. Stöcker, Micropor. Mater. 6 (1996) 235.

[29] J. Xie, M. Huang, S. Kaliaguine, Appl. Surf. Sci. 115 (1997) 157.

[30] J. Tsou, P. Magnoux, M. Guisnet, J.J.M. Órfão, J.L. Figueiredo, Appl. Catal. B: Environ. 51 (2004) 129.

[31] M. Huang, A. Adnot, S. Kaliaguine, J. Catal. 137 (1992) 322.

[32] Q. Li, S.E. Brown, L.J. Broadbelt, J.-G. Zheng, N.Q. Wu, Micropor. Mesopor. Mater. 59 (2003) 105.

[33] D. Brunel, A.C. Blanc, E. Garrone, B. Onida, M. Rocchina, J.B. Nagy, D.J. Macquarrie, Stud. Surf. Sci. Catal. 142 (2002) 1395. 